\title{
Presentación del Tema Central \\ El lenguaje y sus problemas \\ de enseñanza
}

\section{Presentation of the Central Theme The language and its teaching problems}

\author{
Irma Munguía Zatarain \\ Universidad Autónoma Metropolitana Iztapalapa, \\ Ciudad de México, México \\ ORCID: http://orcid.org/0000-0003-1873-905X \\ ISSN: ISSN-OI85-4259; e- ISSN: 2007-9176
} DoI: http://dx.doi.org/I0.28928/revistaiztapalapa/792015/ptc/munguiazataraini

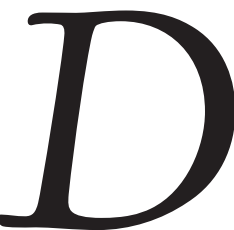

esde hace cuatro décadas, aproximadamente, se escuchan quejas cada vez más constantes de los profesores del medio universitario y de la sociedad en general, respecto de las dificultades de los estudiantes en su habilidad para leer y escribir, como exige el trabajo académico. Incluso, la eficiencia terminal en las universidades ha fracasado, en gran parte porque los alumnos no son capaces de elaborar y redactar una tesis de licenciatura que cumpla con las exigencias propias de un escrito de este tipo, como son la claridad, la coherencia, la argumentación adecuada, la fluidez, el manejo apropiado del léxico, entre otras. Sin duda, la enseñanza de la literatura también ha resentido un retroceso y requiere una profunda revisión para mejorar las prácticas de lectura y apreciación de las obras literarias como expresión artística, como creación, fundamentales en la formación integral de los estudiantes. 
Es evidente que existe un serio y grave problema educativo en el país, en lo que se refiere a la enseñanza de la lengua materna, en general. Es impostergable buscar propuestas de análisis de esta situación, así como esbozar líneas que lleven a un cambio radical en la educación escolar. Y creo que ese cambio debe ser precisamente "radical", dado que en los últimos casi 50 años se han realizado distintos intentos de mejorar el desarrollo de habilidades en los jóvenes, sin ningún éxito significativo, en parte debido a que solo se han introducido cursos remediales, talleres improvisados de redacción y de lectura que en poco o nada han ayudado. Sin duda, falta percatarse de que no se pueden desarrollar habilidades sin un sustento teórico. Es indispensable y urgente repensar y modificar la manera de enseñar el español como lengua materna, y hacer un análisis profundo y sin prejuicios de lo ocurrido en casi medio siglo de impartir una fallida educación — de la manera como se ha hecho-, y encontrar la forma de construir un camino para transitar hacia una salida del problema.

Conviene recordar que la historia de la humanidad está marcada por la reflexión y el estudio de las lenguas; los trabajos y los tratados gramaticales se remontan a Grecia dos siglos antes de nuestra era, y a cuatro siglos si consideramos las investigaciones sobre sánscrito en la antigua India. Siempre ha habido una preocupación central por saber qué son las lenguas, qué las caracteriza y distingue de otras formas de comunicación, por qué hablamos los humanos, cómo lo hacemos y, además, cómo lo debiéramos hacer, sobre todo si nos atenemos a que vivimos en sociedades cada vez más sofisticadas, más exigentes en lo que se refiere al manejo preciso y adecuado del lenguaje. Esta larga tradición - de estudiar gramática en la escuela-, que se extiende por más de dos milenios, se rompió a principios de los años setenta en nuestro país; es decir, se comenzó a abandonar la reflexión sobre la lengua y el estudio explícito y sistemático de la gramática del español, y se sustituyó por cursos de lectura, de redacción y de investigación documental. Pero los resultados son exiguos, como lo han mostrado los diversos exámenes - elaborados con un gran rigor- que se aplican a gran escala en el país, como el examen PISA (por sus siglas en inglés Programme for International Student Assessment), coordinado por la Organización para la Cooperación y el Desarrollo Económicos (OCDE), la Evaluación Nacional de Logro Académico en Centros Escolares (Enlace), el Examen de Calidad y Logro Educativos (eXCAle), el Examen Nacional de Ingreso a la Educación Superior (eXhani-iI), la Prueba de Aptitud Académica (PAA), entre otros.

La temática central del presente número de la revista ofrece la oportunidad de analizar esta compleja situación y enfrenta la problemática desde distintas perspectivas, en cuatro trabajos que aportan algunas líneas para desentrañar lo que ha 
ocurrido en las últimas cuatro décadas y, así, encontrar posibles soluciones a este problema educativo nacional tan apremiante.

En el primer trabajo, titulado "La ausencia de la formación gramatical en la enseñanza del español en México y sus efectos", Irma Munguía hace un breve recorrido histórico para rastrear el origen de los problemas que tienen actualmente los estudiantes universitarios en el desarrollo de sus habilidades para escribir y leer con fluidez y corrección. Plantea que el sistema educativo nacional extravió el camino en su afán de buscar el desarrollo solamente de habilidades, sin ofrecer un conocimiento sistemático sobre gramática y nociones fundamentales de lingüística general. Para ello, presenta un somero análisis de lo que caracterizó a la enseñanza tradicional antes de los años setenta, así como qué rasgos definieron las reformas educativas en 1972-1973 y 1993.

Después de observar el fracaso de estas reformas, propone la recuperación de los contenidos disciplinarios gramaticales — a la luz de las nuevas teorías lingüísticas-, puesto que con ellos sería más factible desempantanar la enseñanza del español y obtener un posible éxito. Munguía parte de la idea de que la reflexión metalingüística que implica el estudio gramatical promovería la conciencia lingüística en los estudiantes, la cual ayudaría a emplear el lenguaje, por lo menos, con mayor control y seguridad; además, daría sustento teórico a los talleres de lectura y de redacción, que se han estado trabajando sin ningún apoyo teórico; la idea de escribir por escribir, sobre cualquier tema, sin un objetivo claro, o de leer por leer sin propósitos específicos - excepto supuestamente para "aprender" estrategias de lectura-, ha llevado a que los estudiantes pierdan el interés por las materias sobre lengua. Y, como consecuencia, los cursos han perdido su rumbo, su sentido, su eficacia. Según la autora, esto se debe a que carecen de contenido real y de sustento teórico.

Con el fin de buscar una solución, la autora habla de la urgencia de contar con una gramática didáctica contemporánea que ayude a trabajar de manera adecuada y gradual aquellos contenidos disciplinarios sobre los que deberían reflexionar los estudiantes en sus doce años de instrucción escolar preuniversitaria, sin que ello implique el abandono de ciertos cursos de redacción y de lectura.

Margarita Galán, en su artículo "Comprensión auditiva”, reflexiona acerca de la gran importancia de la capacidad de escuchar y aprender a partir de los discursos orales a los que se enfrentan los individuos en el medio académico. La información obtenida por esa vía es una fuente de información central; asimismo, desempeña un papel fundamental en el desarrollo de la vida en sociedad. La autora explica cómo en nuestro país no se le ha prestado la suficiente atención al desarrollo de 
esta habilidad; de hecho, señala que no se enseña de manera explícita, ni se la ha evaluado, excepto en el EXHALING. ${ }^{1}$

Termina proponiendo que la comprensión auditiva debiera ser una prioridad de primer orden en el medio escolar, dado que con su adecuado desarrollo podría obtenerse un mayor éxito en las tareas de escribir y leer. Me parece necesario recordar que es a través del oído como el ser humano comienza a enfrentarse a información nueva, desde la más temprana edad, y continúa a lo largo de la vida, a pesar del carácter predominante de la información visual que se ha observado durante las últimas décadas. Sin duda, la comprensión auditiva cumple un papel fundamental en el aprendizaje؛ por esta vía se adquiere la lengua materna, por esta misma vía se aprende en la escuela, en la calle, en los cafés, en las conversaciones con amigos, en conferencias, etcétera.

Ma. del Refugio Pérez, en su artículo "La semántica cognoscitiva como herramienta en el proceso de comprensión lectora", parte del hecho real de que los diferentes cursos de lectura escolares no han sido útiles para enseñar a leer textos académicos, a pesar de que se han hecho varios intentos de cambios, como la implantación del enfoque comunicativo en la década de los noventa. Debido a ello, aboga por el retorno de los contenidos gramaticales en los cursos de español, pero no los tradicionales que se enseñaban antiguamente; propone aprovechar ciertas nociones de semántica léxica, dentro de la llamada lingüística cognoscitiva, pues considera que serían una herramienta fundamental para desarrollar las habilidades de comprensión y producción de textos. Los ejemplos que aporta parecen realmente reveladores y, tal vez, convendría ampliar y profundizar más en esta propuesta.

Finalmente, Martha Elena Munguía, en su trabajo titulado "Los caminos extraviados en la enseñanza de la literatura. Posibles puntos de encuentro", plantea, de manera muy incisiva, el problema de que la enseñanza de la literatura en las escuelas ha sido marginada — tanto como el estudio de la gramática—, pero sobre todo que se ha equivocado la manera de abordarla. Por ejemplo, señala el error reiterativo de motivar a leer solo con la idea de cautivar a los estudiantes con la promesa de que se identificarán con algún personaje, la trama o el autor, con lo cual solamente se ha logrado reducir la lectura del texto literario a un simple fenómeno de "yo he

I El examen de habilidades lingüísticas (EXHALING) fue un proyecto de investigación patrocinado por ANuies, que trató de medir el desarrollo de cuatro habilidades de los estudiantes de primer ingreso en el medio universitario: leer, escuchar, escribir, así como su nivel de conciencia lingüística. Los resultados fueron publicados recientemente en Rosa Obdulia González Robles (coord.), Habilidades lingüisticas de los estudiantes de primer ingreso a las Instituciones de Educación Superior. Área Metropolitana de la Ciudad de México, México, Anuies, 2014. 
vivido o sentido lo mismo"; de esta manera el estudiante pierde la oportunidad de conocer, entender y tal vez admirar y asimilar una visión o mirada nueva, ajena, nunca imaginada, que no es la suya ni la ha conocido nunca, e integrarla de alguna manera en su haber.

La autora habla también del desatino que ha implicado promover en la escuela lecturas solo de textos contemporáneos, lo cual se ha debido, tal vez, a pensar que la literatura del pasado plantea realidades muy distantes y diferentes de las que vivimos en la actualidad, cuando es fácil darnos cuenta de que aquellas realidades remotas serían interesantes de conocer, puesto que así entenderíamos mejor las distintas maneras de interpretar el mundo, de resolver problemas, de actuar, y en esa medida seríamos probablemente mejores intérpretes de la realidad actual. Es notoria la falta de interés de los alumnos en los textos literarios. El artículo advierte sobre la importancia de revisar la metodología y los enfoques que se han empleado, cuyo objetivo debiera ser guía para estos cursos: la formación de individuos críticos, conscientes de su realidad, lo cual se puede lograr, sin duda, a partir de una mejor práctica en el análisis literario escolar.

Como puede verse, en líneas generales, el tema central de este número gira en torno a propuestas encaminadas a plantear la necesidad de un cambio profundo $y$ radical en los contenidos, marcos teóricos y metodologías en los planes y programas de educación preuniversitaria, con el fin de superar el estancamiento y la frustración en los que se ha atascado la enseñanza del lenguaje; se ha mostrado repetidamente en varios estudios que los talleres o cursos de redacción y de lectura no son suficientes. Sin duda, creo que hace falta salir de la inercia de realizar investigaciones que solo evalúan la pésima formación que tenemos pero no proponen nada, así como la de tratar de remediar la situación con más cursos de redacción. Insisto en que hace falta considerar con seriedad lo que podría aportar la reflexión metalingüística en el sistema educativo, a partir de bases teóricas que la lingüística contemporánea ofrece; han transcurrido ya casi cinco décadas y el fracaso escolar es cada vez más escandaloso y preocupante, por lo que es hora de detenernos, revisar cuidadosamente lo que se ha hecho y construir nuevas propuestas de enseñanza que se despojen de los esquemas arraigados en la sociedad, como son la desconfianza y el rechazo hacia los estudios gramaticales, ${ }^{2}$ el desprecio por la normatividad lingüística, ${ }^{3}$ la visión

2 Tal vez este repudio tiene que ver con miedo, desconocimiento o ignorancia completa de lo que es estudiar gramática.

3 Quienes desprecian la normatividad y defienden el empleo del lenguaje libre de reglas y de normas, al mismo tiempo caen en la contradicción de rechazar a las personas que se expresan mal y, además, cuando escriben, se sujetan a esas normas. 
simplista y empobrecida de que todo contenido que se estudie debe ser útil y tener una aplicación inmediata, entre muchos más.

En la actualidad, la realidad nacional reclama urgentemente estudios innovadores en estos campos, es decir, en la enseñanza del español como lengua materna, así como en la iniciación en el análisis literario, para poder tener éxito en la tarea de formar estudiantes más conscientes y exitosos en el manejo de su lengua, así como más capaces de apreciar y valorar la escritura artística. 